\title{
Depression and Structural Factors Are Associated With Symptoms in Patients of Irritable Bowel Syndrome With Diarrhea
}

\author{
Jia Lu, ${ }^{1}$ Lili Shi, ${ }^{2}$ Dan Huang, ${ }^{3}$ Wenjuan Fan, ${ }^{1}$ Xiaoqing Li, ${ }^{1}$ Liming Zhu, ${ }^{1}$ Jing Wei, ${ }^{2}$ and Xiucai Fang ${ }^{1 *}$ \\ Departments of ${ }^{1}$ Gastroenterology and ${ }^{2}$ Psychological Medicine, Peking Union Medical College Hospital, Chinese Academy of Medical Sciences \\ and Peking Union Medical College, Beijing, China; and ${ }^{3}$ Department of Gastroenterology, The People's Hospital of Guangxi Zhuang Autonomous \\ Region, Nanning, China
}

\section{Background/Aims}

A strong correlation between depression and irritable bowel syndrome with diarrhea (IBS-D) has been identified. The aim of this study is to identify the correlations among depression, structural factors, gastrointestinal (GI) and extra-GI symptoms, and efficacy of neuromodulators in patients with IBS-D.

\section{Methods}

Patients meeting the Rome III Diagnostic Criteria for IBS-D were enrolled. The intestinal symptoms and psychological states were evaluated using IBS-specific symptom questionnaires and Hamilton Depression Rating Scale.

\section{Results}

In total, 410 patients with IBS-D were enrolled, 28.8\% (118/410) had comorbid depression. Patients with depression did not readily experience improvement in abdominal pain/discomfort after defecation, and had a higher prevalence of passing mucus, overlapping functional dyspepsia, and extra-Gl symptoms. The structural factor "mental disorders" significantly correlated with main bowel symptom score and degree of pre-defecation abdominal pain/discomfort. No structural factor significantly correlated with bowel movements or stool form. Patients who had passing mucus, overlapping functional dyspepsia and extra-Gl painful symptoms have higher score of "anxiety/somatization." Patients with sexual dysfunction have higher score of "retardation symptoms." In total, 28.3\% of patients with IBS-D were prescribed neuromodulators. Baseline scores of "anxiety/somatization" and "retardation symptoms" positively correlated with improvement of diarrhea after paroxetine, and "sleep disturbances" positively correlated with improvement of abdominal pain/discomfort and diarrhea after mirtazapine.

\section{Conclusions}

Comorbid depression and higher scores of structural factors might aggravate Gl and extra-Gl symptoms other than bowel movements and stool form. Structural factors of Hamilton Depression Rating Scale correlated with efficacy of paroxetine and mirtazapine in patients with IBS-D.

(J Neurogastroenterol Motil 2020;26:505-513)

\section{Key Words}

Depression; Irritable bowel syndrome; Neuromodulators

Received: August 31, 2019 Revised: April 28, 2020 Accepted: June 1, 2020

(.) This is an Open Access article distributed under the terms of the Creative Commons Attribution Non-Commercial License (http://creativecommons. org/licenses/by-nc/4.0) which permits unrestricted non-commercial use, distribution, and reproduction in any medium, provided the original work is properly cited.

${ }^{*}$ Correspondence: Xiucai Fang, MD

Department of Gastroenterology, Peking Union Medical College Hospital, Chinese Academy of Medical Sciences and Peking Union Medical College, 1\# Shuaifuyuan, Dongcheng District, Beijing 100730, China

Tel: +86-10-6915-6892/+86-1343-913-6086, Fax: +86-10-6915-1963, E-mail: fangxiucai2@aliyun.com

Jia Lu and Lili Shi equally contributed to this work. 


\section{Introduction}

Irritable bowel syndrome (IBS) is one of the most common functional gastrointestinal (GI) disorders and is characterized by abdominal pain/discomfort and altered bowel habits. The estimated global prevalence of IBS is $8.8 \%$. $^{1}$ In China, the prevalence is about $6.5 \%$ and IBS with diarrhea (IBS-D) is the most common subtype. ${ }^{2}$ The pathogenesis of IBS involves multiple factors and mechanisms, including dysmotility, visceral hypersensitivity, gut microbiome alterations, and abnormal brain-gut interaction. ${ }^{3}$ Psychological factors also play an important role. ${ }^{4}$ Patients with IBS frequently have comorbid depression (prevalence of up to $50 \%)^{5}{ }^{5}$ Patients with mental disorders also have increased risk of IBS. ${ }^{6}$ Psychotherapy and antidepressants (currently called neuromodulators) improve bowel symptoms while significantly improving the mental state of patients with IBS, ${ }^{7-9}$ even those with refractory IBS for which traditional treatments were ineffective. ${ }^{10}$ The Hamilton Depression Rating Scale (HAMD) has been used to evaluate the depressive state of patients with IBS in many studies. ${ }^{11}$ Studies on depression suggest that the structural factors of the HAMD might reflect the characteristics of depression; they also correlate with the severity of depression, and predict the efficacy of neuromodulators. ${ }^{12,13}$ However, studies on the features of comorbid depression, the indications and choices of neuromodulators for IBS are insufficient. Therefore, the present study provides clinical evidence for treating IBS-D through evaluation of patients' depressive state and analysis of the correlations between depression features reflected by structural factors, GI and extra-GI symptoms as well as the efficacy of neuromodulators in IBS-D patients.

\section{Materials and Methods}

\section{Study Population}

Patients aged 18 to 70 years who met the Rome III criteria for IBS- ${ }^{14}$ were consecutively recruited from the gastroenterology clinics from July 2009 to May 2017. Patients with organic GI diseases, connective tissue diseases, and metabolic diseases as confirmed by laboratory and endoscopy examinations in the last year were excluded. ${ }^{15}$ All patients were informed about the study, after which they provided either written or verbal consent to participate. This study was approved by the Ethics Committee of hospital (Approval No. S-234).

\section{Instrumental Evaluation}

GI symptoms were assessed using the IBS symptom questionnaire, ${ }^{16}$ and included demographic information, main intestinal symptoms, defecation-related symptoms, and extra-intestinal symptoms, which were completed in a face-to-face interview. The questionnaire included demographic information, main bowel symptoms, defecation-related symptoms, upper GI symptoms, extraGI symptoms, psychological state, and sleep state. The main bowel symptoms (frequency and degree of pre-defecation abdominal pain/ discomfort, bowel movements and stool form during symptom onset, and degree of improvement of abdominal pain/discomfort after defecation) were scored as previously described, ${ }^{16}$ each with a 4-point scale ranging from 0 (none) to 3 (onset every day or symptom in severe or less relief) and the possible overall scale of $15{ }^{16}$ Bowel movements and stool form in the interictal period were scored by the same standard and Bristol stool form scale. ${ }^{14}$ Gastroesophageal reflux disease (GERD) was diagnosed according to the Montreal consensus. ${ }^{17}$ Functional dyspepsia (FD) fulfilled the Rome III diagnostic criteria. ${ }^{14}$ Depressive symptoms were assessed using the 17 -item HAMD, ${ }^{12}$ which was translated to Chinese version in 1984 and had been validated to be widely used in China. ${ }^{18,19}$

The HAMD was administered by 3 specially trained professionals through conversation and observation. Depression states were defined as follows: normal (total HAMD score of $\leq 7$ ), possible depression (8-16), mild depression (17-23), and moderate to severe depression $(\geq 24)$. The 17 items were grouped into 5 structural factors: "anxiety/somatization" (including items of psychic anxiety, somatic anxiety, GI symptoms, general somatic symptoms, hypochondriasis [self-absorption, ie, bodily, hypochondriacal delusions, etc], and insight), "mental disorders" (including items of guilt, suicide, and agitation), "retardation symptoms" (including items of depressed mood, work and interests, retardation, and genital symptoms), "sleep disturbances" (including items of initial insomnia, middle insomnia, and delayed insomnia), and "weight loss." ${ }^{12,20,21}$ The patients also completed the Hamilton Anxiety Rating Scale (HAMA) (data not shown).

Some patients with IBS-D were prescribed neuromodulators according to the following criteria: patients have comorbid depression and/or anxiety, refractory IBS, for which traditional treatments (ie, antispasmodic, loperamide, probiotics, and traditional Chinese medicine) were ineffective and discontinued. A visual analog scale (VAS) was used to evaluate improvements in the following 4 symptoms after $\geq 1$ month of treatment with neuromodulators: emotion, sleep quality, degree of pre-defecation abdominal pain/discomfort, 
and diarrhea. Improvement was defined as follows: no improvement (no change in symptoms or VAS score), mild improvement (obvious symptoms that significantly impair daily life with a 1-3 points decrease in VAS score), moderate improvement (symptoms that impair daily life with a 4-7 points decrease), and significant improvement or full mitigation (slight symptoms or recovery with an 8-10 points decrease).

\section{Statistical Methods}

Data were analyzed using SPSS 23.0 software (IBM Corporation, Armonk, NY, USA). Continuous variables are presented as mean \pm standard deviation or median (interquartile range [IQR]), and categorical variables are presented as a ratio. Continuous variables were analyzed using the independent two-sample $t$ test and correlation analysis. Categorical variables were compared using the chi-square test and nonparametric test. Correlation analysis was performed using Spearman rank correlation analysis. A $P$-value of $<0.05$ was considered statistically significant.

\section{Results}

\section{Demographic and Clinical Characteristics}

In total, 410 patients with IBS-D were enrolled (250 male, 160 female; sex ratio, 1.56:1). Their mean age was $41.9 \pm 11.3$ years (range, 18-68 years). The mean disease course of IBS-D was 4.5 (IQR $[2.0,10.0])$ years.

\section{Depressive State and Characteristics Reflected by Structure Factors of Patients With Irritable Bowel Syndrome}

The mean HAMD score was $13.2 \pm 5.9$, and there were no significant differences in the HAMD scores assessed by 3 investigators $(P>0.05)$. In total, $28.8 \%(118 / 410)$ of patients had comorbid depression (total HAMD score of $\geq 17) ; 25.1 \%(103 / 410)$ had mild and $3.7 \%(15 / 410)$ had moderate to severe depression. In all patient groups, the anxiety/somatization score was the highest among the terms of the structural factors of the HAMD (Table 1), $71.7 \%$ (294/410) of patients had self-absorption (bodily); $20.9 \%$ (86/410) were troubled by hypochondriacal delusions, of whom $41.9 \%$ (36/86) without depression. In factors of mental disorders, $34.4 \%(141 / 410)$ of patients felt guilty, of whom $60.3 \%(85 / 141)$ without depression; $1.2 \%$ (5/410) had negative thoughts (suicidal ideation), of whom all had comorbid depression.

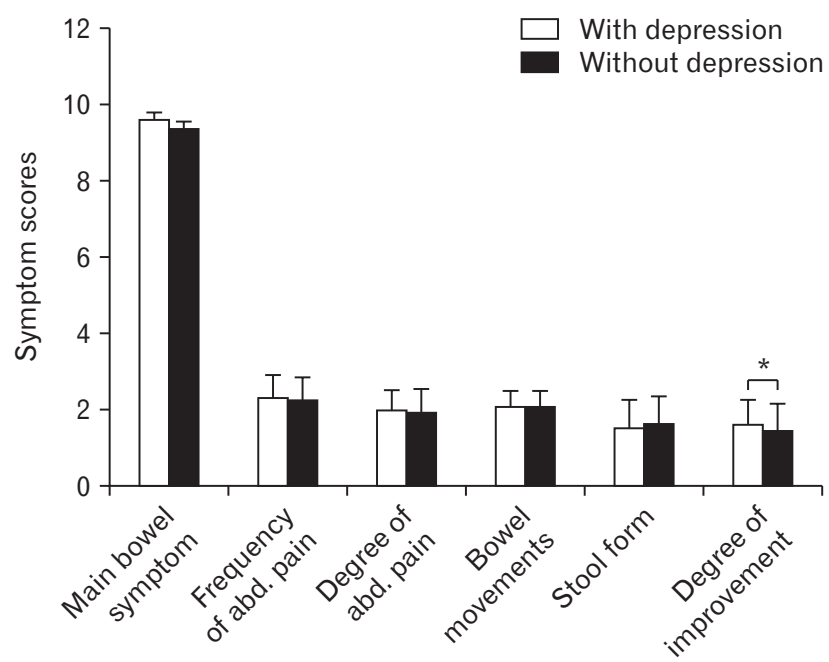

Figure 1. Scores of main bowel symptoms in irritable bowel syndrome with diarrhea patients with and without depression. The score for the degree of improvement in abdominal pain/discomfort after defecation in patients with depression is higher than that in patients without depression. ${ }^{*} P<0.05$. abd. pain, abdominal pain/discomfort.

Table 1. Scores of Hamilton Depression Rating Scale and Structural Factors of Patients With Irritable Bowel Syndrome With Diarrhea

\begin{tabular}{|c|c|c|c|c|}
\hline $\begin{array}{c}\text { HAMD and } \\
\text { structural factors }\end{array}$ & $\begin{array}{l}\text { All patients } \\
(\mathrm{N}=410)\end{array}$ & $\begin{array}{l}\text { Patients with depression } \\
\qquad(\mathrm{n}=118)\end{array}$ & $\begin{array}{c}\text { Patients without } \\
\text { depression }(n=292)\end{array}$ & $P$-value ${ }^{\mathrm{a}}$ \\
\hline HAMD & $13.2 \pm 5.9$ & $20.4 \pm 3.4$ & $10.3 \pm 3.8$ & $<0.001$ \\
\hline Anxiety/somatization & $5.8 \pm 2.2$ & $7.9 \pm 1.6$ & $4.9 \pm 1.8$ & $<0.001$ \\
\hline Mental disorders & $1.3 \pm 1.3$ & $2.4 \pm 1.4$ & $0.9 \pm 0.9$ & $<0.001$ \\
\hline Retardation symptoms & $2.5 \pm 1.9$ & $4.5 \pm 1.6$ & $1.7 \pm 1.3$ & $<0.001$ \\
\hline Sleep disturbances & $1.9 \pm 1.8$ & $3.1 \pm 1.8$ & $1.5 \pm 1.6$ & $<0.001$ \\
\hline Weight loss & $0.7 \pm 0.7$ & $1.0 \pm 0.7$ & $0.5 \pm 0.7$ & $<0.001$ \\
\hline
\end{tabular}

${ }^{\mathrm{a}}$ Comparison between patients with depression and without depression.

HAMD, Hamilton Depression Rating Scale.

Data present as mean $\pm \mathrm{SD}$. 
Comparing to the patients without depression, we found the scores of HAMD and 5 structural factors were significant higher in patents with depression (Table 1).

\section{Correlation of Hamilton Depression Rating Scale, Structural Factors and Main Bowel Symptoms}

The main bowel symptom score was $9.5 \pm 1.5$. There were no significant differences in the scores of main bowel symptom, frequency and degree of pre-defecation abdominal pain/discomfort, bowel movements and stool form during symptom onset between patients with depression and without depression, but those with depression attained a higher score for the degree of improvement after defecation ( $t=2.136, P=0.033$ ), which indicated less improvement in the intensity of abdominal pain/discomfort after defecation (Fig. 1).

The HAMD score positively correlated with the main bowel symptom score, scores of pre-defecation abdominal pain/discomfort and degree of improvement of abdominal pain/discomfort after defecation (all $P<0.05$ ). The mental disorders score positively correlated with the main bowel symptom score, scores of degree of predefecation abdominal pain/discomfort and degree of improvement of abdominal pain/discomfort after defecation (all $P<0.05$ ). The anxiety/somatization and sleep disturbances scores also positively correlated with the scores of degree of improvement of abdominal pain/discomfort after defecation (all $P<0.05$ ). There was no significant correlation of any structural factor with bowel movements and stool form either during symptom onset or in the interictal period (Fig. 2).

\section{Correlation of Hamilton Depression Rating Scale, Structural Factors and Defecation-related Symptoms}

The prevalence of abdominal bloating, urgency, straining, incomplete defecation, and anorectal pain was not significantly different between patients with and without depression, but those with depression had a higher prevalence of passing mucus $(73.7 \%$ vs $60.3 \%, \chi^{2}=6.62, P=0.010$ ) (Fig. 3). The HAMD, anxiety/somatization, and retardation symptoms scores were higher in patients who did than did not pass mucus $(P<0.05)$ (Table 2).

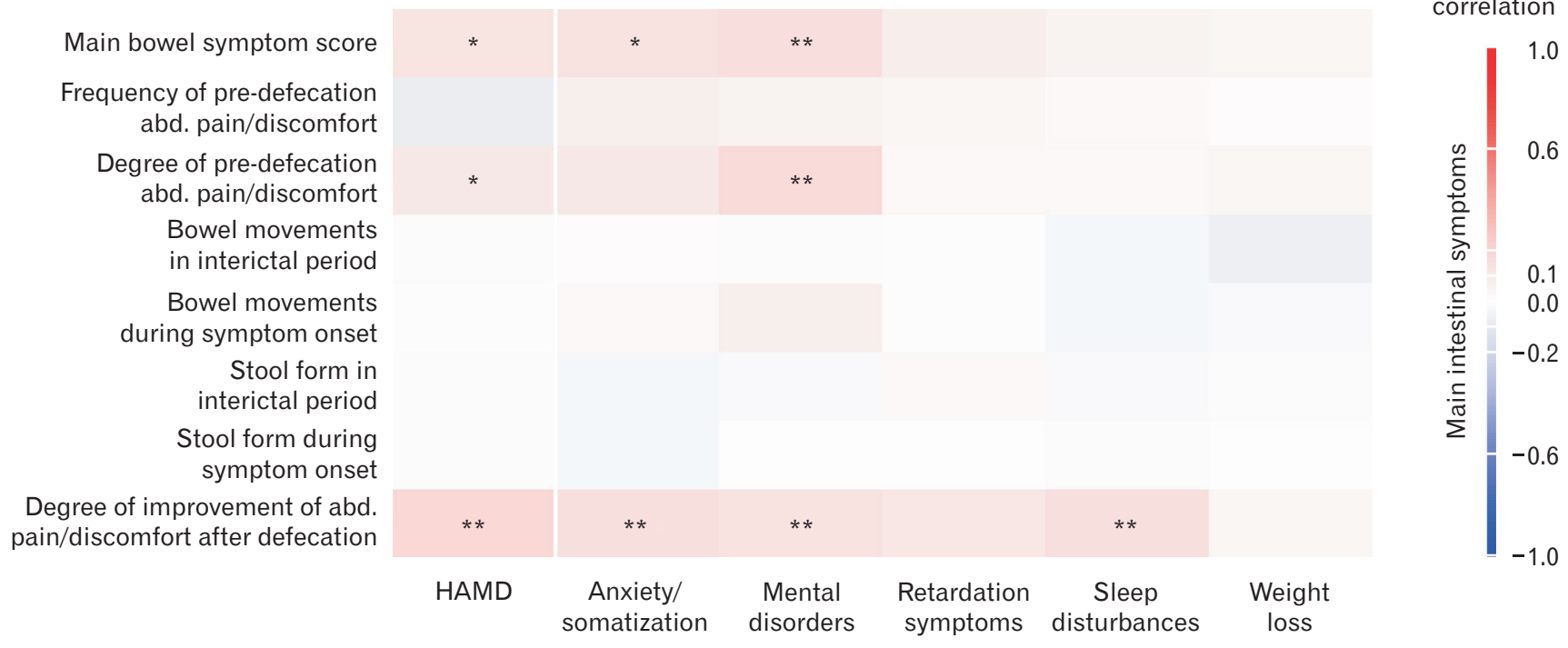

Figure 2. Heat map of the Spearman rank correlations of Hamilton Depression Rating Scale (HAMD) and structural factor scores with the main bowel symptoms of irritable bowel syndrome with diarrhea. Score of HAMD positively correlated with the scores of main bowel symptom score $(r$ $=0.127, P=0.010)$, degree of pre-defecation abdominal pain/discomfort $(r=0.101, P=0.040)$ and improvement of abdominal pain/discomfort after defecation $(r=0.183, P<0.001)$. Score of mental disorders positively correlated with the scores of main bowel symptom $(r=0.154, P$ $=0.002)$, degree of pre-defecation abdominal pain/discomfort $(r=0.172, P<0.001)$ and degree of improvement of abdominal pain/discomfort after defecation $(r=0.135, P=0.006)$. Scores of anxiety/somatization and sleep disturbances also positively correlated with the score of degree of improvement of abdominal pain/discomfort after defecation $(r=0.144, P=0.004 ; r=0.150, P=0.002)$. ${ }^{*} P<0.05$, ** $P<0.01$. abd., abdominal. 


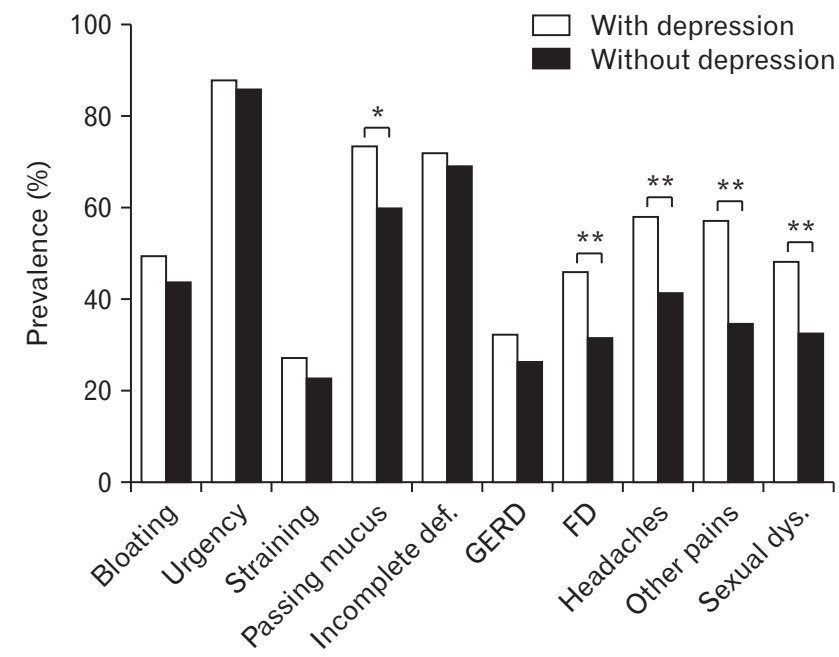

Figure 3. Prevalence of defecation-related symptoms, upper gastrointestinal symptoms, and extra-gastrointestinal symptoms in irritable bowel syndrome with diarrhea (IBS-D) patients with and without depression. The prevalence of passing mucus, overlap with functional dyspepsia (FD), headache, pain in other areas, and sexual dysfunction was significantly higher in patients with depression. ${ }^{\star} P<0.05,{ }^{*} P$ $<0.01$ comparing to patients without depression. GERD, gastroesophageal reflux disease; def., defecation; dys., dysfunction.

\section{Correlation of Hamilton Depression Rating Scale, Structural Factors and Overlapping Upper Gastrointestinal Symptoms}

The prevalence of overlapping GERD was not significantly different between patients with and without depression, but those with depression had a higher prevalence of $\mathrm{FD}(46.6 \%$ vs $32.2 \%$, $\chi^{2}=7.55, P=0.006$ ) (Fig. 3). The HAMD, anxiety/somatization, retardation symptoms, mental disorders, and sleep disturbances scores were higher in patients with than without FD $(P<0.05)$ (Table 2).

\section{Correlation of Hamilton Depression Rating Scale, Structural Factors and Extra-gastrointestinal Symptoms}

Patients with IBS-D had multiple extra-GI symptoms. The prevalence of headache, pain in other areas (including muscles and joints), and sexual dysfunction was higher in patients with than without depression $\left(\chi^{2}=9.02, P=0.003 ; \chi^{2}=17.06, P<0.001\right.$; $\chi^{2}=8.01, P=0.005$ ) (Fig. 3). The HAMD, anxiety/somatization, and retardation symptoms scores were higher in patients with than without headache, pain in other areas and sexual dysfunction $(P$ $<0.05$ ) (Table 2).

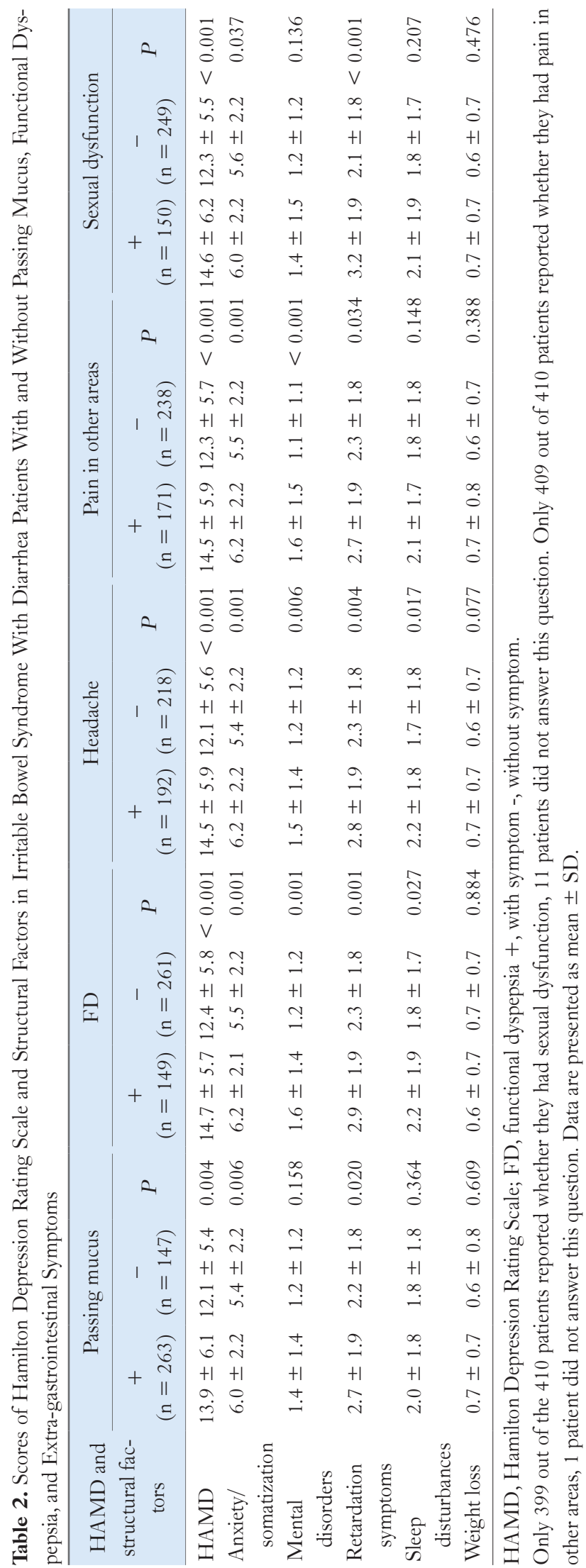




\section{Correlation of Hamilton Depression Rating Scale, Structural Factors and Efficacy of Neuromodulators}

In total, $116(28.3 \%)$ patients with IBS-D were prescribed neuromodulators for $\geq 1$ month, and $16.4 \%$ (19/116) dropped out during follow-up. The mean HAMD score of patients who were prescribed neuromodulators was $15.2 \pm 5.9$. A total of $41.4 \%$ (48/116) had comorbid depression; $34.5 \%$ (40/116) had mild and $6.9 \%(8 / 116)$ had moderate to severe depression. Additionally, $70.7 \%$ (82/116) had comorbid anxiety as assessed by the HAMA, and $18.0 \%(21 / 116)$ had no depression or/and anxiety.

Among the 97 patients with complete follow-up data, the most commonly used neuromodulators were paroxetine $(\mathrm{n}=52,20-40$ $\mathrm{mg} /$ day), mirtazapine ( $\mathrm{n}=50,15-45 \mathrm{mg} /$ day), and flupentixol/melitracen ( $\mathrm{n}=17,10-20 \mathrm{mg} /$ day), accounting for $88.7 \%$ of patients (86/97, including those undergoing sequence or augmentation therapy). Other neuromodulators included sertraline, escitalopram, venlafaxine, and fluoxetine. The mean treatment duration was 5.0 (IQR [2.0, 12.4]) months; 98.8\% (85/86) reported different degrees of improvement in emotion, sleep quality, pre-defecation
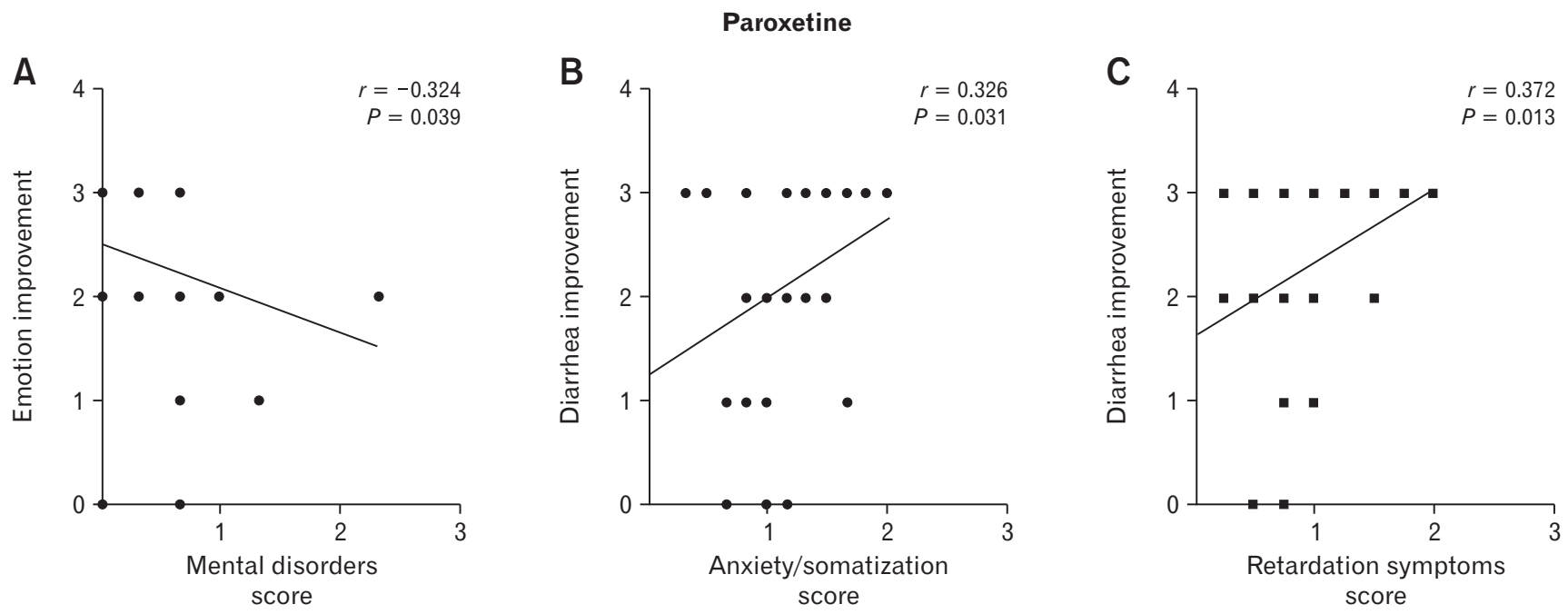

Mirtazapine
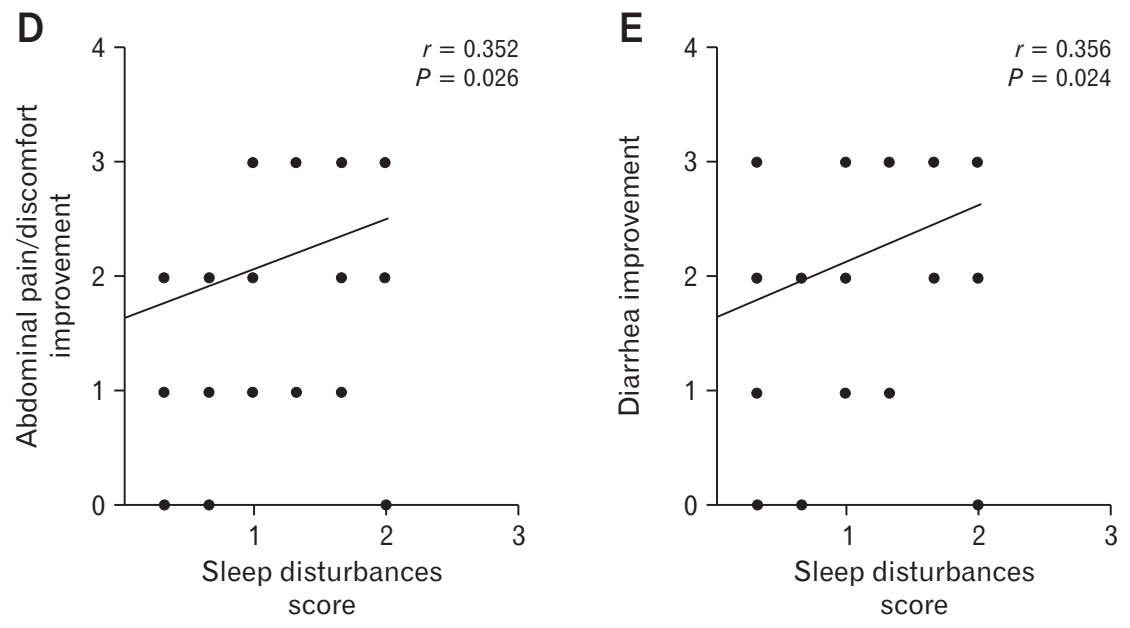

Figure 4. Correlations between structural factors of Hamilton Depression Rating Scale and the efficacy of neuromodulators in patients with irritable bowel syndrome with diarrhea. (A) The baseline mental disorders score negatively correlated with improvements in emotions. (B, C) The scores of anxiety/somatization and retardation symptoms positively correlated with improvement in diarrhea after paroxetine therapy. (D, E) The baseline sleep disturbances score positively correlated with improvements in defecation-related abdominal pain/discomfort and diarrhea after mirtazapine therapy. 
abdominal pain/discomfort, and diarrhea after $\geq 1$ month of neuromodulators, while $15.1 \%$ (13/86) of patients reported either significant improvement or full recovery of all symptoms. The degree of emotional improvement positively correlated with improved sleep quality and decreased abdominal pain/discomfort and diarrhea $(r=$ 0.656, $P<0.001 ; r=0.540, P<0.001 ; r=0.459, P<0.001)$. The baseline mental disorders score negatively correlated with improvements in emotion $(r=-0.324, P=0.039)$, and the anxiety/ somatization and retardation symptoms scores positively correlated with improvement of diarrhea after paroxetine therapy $(r=0.326$, $P=0.031 ; r=0.372, P=0.013)$. The baseline sleep disturbances score positively correlated with improvements in abdominal pain/ discomfort and diarrhea after mirtazapine therapy $(r=0.352, P=$ $0.026 ; r=0.356, P=0.024$ ) (Fig. 4). No structural factor showed a significant correlation with improvement of the above symptoms after flupentixol/melitracen therapy.

\section{Discussion}

Patients with IBS commonly have comorbid depression, ${ }^{11,22,23}$ little attention is given to the characteristics of comorbid depression and the relationship with bowel symptoms and the efficacy of neuromodulators in IBS. This study showed that patients of IBS-D with depression did not readily experience improvements in abdominal pain/discomfort after defecation, and had a higher prevalence of passing mucus, overlapping FD and extra-GI symptoms. Patients with passing mucus, FD and extra-GI symptoms had higher scores of anxiety/somatization and retardation symptoms. The anxiety/ somatization and retardation symptoms scores positively correlated with improvement of diarrhea after paroxetine therapy, and sleep disturbances score positively correlated with improvements in abdominal pain/discomfort and diarrhea after mirtazapine therapy.

HAMD has good reliability and validity, it comprises of 17 items and is grouped into 5 structural factors (ie, anxiety/somatization, mental disorders, retardation symptoms, sleep disturbances, and weight loss) by Cleary and Guy. ${ }^{20}$ Factor analysis (ie, structural factors) can identify the characteristics of depression in patients with depression and predict the outcomes of antidepressant therapy. ${ }^{12,13}$ Anxiety/somatization comprises of 6 items: psychic anxiety, somatic anxiety, GI symptoms, general somatic symptoms, hypochondriasis, and insight. A cross-sectional study in major depressive disorders (MDD) showed that patients with an anxiety/somatization score of $\geq 7$ had more pain symptoms, impaired functions, and reduced quality of life, ${ }^{24}$ the present study showed IBS-D patients with depression also have higher anxiety/somatization (
Hypochondriasis is an important item of the anxiety/somatization factor in HAMD. Studies conducted with the Minnesota Multiphasic Personality Inventory found that hypochondriasis was related to abdominal pain, ${ }^{25}$ and significant impaired the quality of life in IBS patients. ${ }^{26}$ In present study, $41.9 \%$ patients with hypochondriasis do not have depression, suggesting that hypochondriasis is an important and independent structural factor of depression for patients with IBS-D. IBS patients with hypochondriasis are under the illusion of having severe organic diseases which result in seeking repeated examinations and wasting many medical resources. Therefore, in clinical practice, more attention should be paid to the psychological characteristics reflected by hypochondriasis of the HAMD in IBS patients, especially for those without depression via regular evaluation.

Mental disorders comprises 3 items: guilt, suicide, and agitation. This factor most strongly correlated with the severity of abdominal pain/discomfort in patients with IBS-D. Guilt in patients with IBS arises from the burden of GI symptoms to the family; ${ }^{27}$ suicidal ideation is related to hopelessness because of symptom severity, interference with life, and inadequacy of treatment. ${ }^{28}$ Thus, for IBS patients with severe abdominal pain, more attention should be paid to mental disorders, especially guilt and suicidal ideation while interpreting the report of HAMD. Patients with suicidal tendencies should be referred to psychological professionals as early as possible.

Retardation symptoms is the core factor of depression and includes 4 items: depressed mood, work and interests, retardation, and genital symptoms. In the present study, more than one-third of patients with IBS-D had sexual dysfunction, and these patients scored higher in retardation symptoms. Genital symptoms had no association with the severity of intestinal symptoms of IBS, and patients with sexual dysfunction were more likely to experience more severe depression, ${ }^{29}$ indicating that depression may affect genital symptoms which is independent of intestinal symptoms of IBS. Doctors must place more focus on genital symptoms and retardation symptoms of the HAMD among patients with IBS-D.

Relative to the above structural factors, sleep disturbances and weight loss had relatively limited correlations with GI and extra-GI symptoms in IBS-D. Self-reported sleep disturbances are common in people with IBS. $^{30}$ A recent systematic review showed that sleep disturbances increased visceral hypersensitivity and aggravated the severity and frequency of GI symptoms in patients with IBS, and this indicated that improvement of sleep quality would be helpful to the relief of IBS symptoms. ${ }^{30}$

In general, comorbid depression and its structural factors 
mainly correlated with the sensory symptoms of IBS-D, but not correlated with bowel movements or stool form. Bowel movements and stool form in patients with IBS are commonly believed to be directly correlated with intestinal motility and secretory function. ${ }^{31}$

The efficacy of neuromodulators is related to the mechanism of neuromodulators and the characteristics of depression. Among patients with MDD, early changes in the items of GI symptoms in anxiety/somatization factor can predict the remission (HAMD score of $<8$ ) with fluoxetine therapy. ${ }^{32}$ A high anxiety/somatization score was a predictor of ineffective treatment with duloxetine in patients with MDD. ${ }^{33}$ Few studies to date have focused on IBS. In the present study, IBS patients treated with paroxetine showed a significant improvement of both psychiatric and GI symptoms, which is similar to the current publication. ${ }^{7}$ Baseline anxiety/somatization and retardation symptoms positively correlated with improvement in diarrhea after paroxetine therapy, indicating that paroxetine might be a preferential selection for IBS-D patients with high scores in anxiety/somatization, retardation symptoms, and with severe diarrhea. Sleep disturbances positively correlated with improvement in abdominal pain/discomfort and diarrhea after mirtazapine therapy, which is consistent with pharmacodynamic features of mirtazapine ${ }^{34}$ and our clinical experience.

In this study, $18.0 \%$ of patients who were prescribed neuromodulators did not have comorbid depression or anxiety, and the indication for neuromodulatory therapy focused on their refractory intestinal symptoms; however, nearly all patients reported various degrees of improvements in bowel or mental symptoms. A recent report from the Rome Foundation working team showed that tricyclic neuromodulators can reduce diarrhea, serotonin noradrenalin reuptake inhibitors have the potential to improve pain, and the selective serotonin reuptake inhibitors can be helpful for pain, bloating, and constipation in patients with IBS, even regardless of coexisting anxiety or depression. ${ }^{35}$ These suggest that neuromodulators might have a direct effect on the GI system in patients with IBSD. ${ }^{36}$ Therefore, for patients who have severe intestinal symptoms even with low scores on the depression and anxiety scales, proper selection of neuromodulators may lead to better treatment effects.

There are some limitations. First, this study is a retrospective analysis. Second, the neuromodulators were prescribed by the professionals according to individual situations, without uniform enrolling criteria, doses or duration, these individualized strategies might explain that the overall efficacy of neuromodulators is higher than results of randomized controlled trials. ${ }^{8,35}$ Third, the efficacies were scaled according to the medical records during analysis. We believe this report from "real world" could be helpful for physi- cians to screen those patients being benefit from neuromodulatory therapy.

In conclusion, IBS-D patients with comorbid depression have less improvement of abdominal pain/discomfort after defecation, a higher prevalence of passing mucus, and overlapping FD and extra-GI symptoms. Focusing on structural factors in the HAMD could identify the psychological characteristics and the association with GI and extra-GI symptoms in patients with IBS-D, and the factors of anxiety/somatization and retardation symptoms or sleep disturbances correlated with the efficacy of paroxetine or mirtazapine in patients IBS-D, which might be helpful for optimizing neuromodulators and improving the overall efficacies for IBS patients.

Acknowledgements: We thank Dr Haiyu Pang, for her consulting assistance in statistical analysis. The abstract was presented at Digestive Disease Week 2018 (Jia Lu, Lili Shi, Dan Huang, et al. Anxiety, depression and structural factors aggravate on gastrointestinal symptoms in patients with irritable bowel syndrome with diarrhea. Gastroenterology 2018;154:S977).

Financial support: This work was supported by the Program of International S \& T Cooperation of the Ministry of Science and Technology of the People's Republic of China (Grant No. 2014DFA31850), and The National Natural Science Foundation of China (Grant No. 81870379).

\section{Conflicts of interest: None.}

Author contributions: Jia Lu and Lili Shi analyzed the data and wrote the manuscript; Dan Huang and Wenjuan Fan collected the data and planned the study; Xiaoqing Li, Liming Zhu, and Jing Wei consulted with the patients; and Xiucai Fang designed the study, consulted with the patients, and critically revised the manuscript.

\section{References}

1. Sperber AD, Dumitrascu D, Fukudo S, et al. The global prevalence of IBS in adults remains elusive due to the heterogeneity of studies: a Rome foundation working team literature review. Gut 2017;66:1075-1082.

2. Zhang L, Duan L, Liu Y, et al. [A meta-analysis of the prevalence and risk factors of irritable bowel syndrome in Chinese community.] Zhonghua Nei Ke Za Zhi 2014;53:969-975. [Chinese]

3. Hungin AP, Becher A, Cayley B, et al. Irritable bowel syndrome: an integrated explanatory model for clinical practice. Neurogastroenterol Motil 2015;27:750-763.

4. Wu JC. Psychological co-morbidity in functional gastrointestinal disorders: epidemiology, mechanisms and management. J Neurogastroenterol 
Motil 2012;18:13-18.

5. Kibune Nagasako C, Garcia Montes C, Silva Lrena SL, Mesquiita MA. Irritable bowel syndrome subtypes: clinical and psychological features, body mass index and comorbidities. Rev Esp Enferm Dig 2016;108:5964.

6. Maguen S, Madden E, Cohen B, Bertenthal D, Seal K. Association of mental health problems with gastrointestinal disorders in Iraq and Afghanistan veterans. Depress Anxiety 2014;31:160-165.

7. Stasi C, Caserta A, Nisita C, et al. The complex interplay between gastrointestinal and psychiatric symptoms in irritable bowel syndrome: a longitudinal assessment. J Gastroenterol Hepatol 2019;34:713-719.

8. Ford AC, Quigley EM, Lacy BE, et al. Effect of antidepressants and psychological therapies, including hypnotherapy, in irritable bowel syndrome: systematic review and meta-analysis. Am J Gastroenterol 2014;109:1350-1365

9. Keefer L, Palsson OS, Pandolfino JE. Best practice update: incorporating psychogastroenterology into management of digestive disorders. Gastroenterology 2018;154:1249-1257.

10. Srivastava P, Bhad R, Sharma P, Varshney M, Sharan P. Successful management of difficult-to-treat irritable bowel syndrome incorporating a psychological approach. Natl Med India 2015;28:188-189.

11. Zhang QE, Wang F, Qin G, et al. Depressive symptoms in patients with irritable bowel syndrome: a meta-analysis of comparative studies. Int J Bio Sci 2018;14:1504-1512.

12. Hamilton M. A rating scale for depression. J Neurol Neurosurg Psychiatry 1960;23:56-62.

13. Fava M, Rush AJ, Alpert JE, et al. Difference in treatment outcome in outpatients with anxious versus nonanxious depression: a STAR*D report. Am J Psychiatry 2008;165:342-351.

14. Drossman DA. The functional gastrointestinal disorders and the rome III process. Gastroenterology 2006;130:1377-1390.

15. Fan WJ, Xu D, Chang M, et al. Predictors of healthcare-seeking behavior among Chinese patients with irritable bowel syndrome. World J Gastroenterol 2017;23:7635-7643.

16. Zhu L, Huang D, Shi L, et al. Intestinal symptoms and psychological factors jointly affect quality of life of patients with irritable bowel syndrome with diarrhea. Health Qual Life Outcomes 2015;13:49.

17. Vakil N, van Zanten SV, Kahrilas P, Dent J, Jones R. The Montreal definition and classification of gastroesophageal reflux disease: a global evidence-based consensus. Am J Gastroenterol 2006;101:1900-1920.

18. Tang YH, Zhang MY. [Hamilton Depression Scale (HAMD).] Shanghai Archives of Psychiatry 1984;2:61-64. [Chinese]

19. Zhao JP, Zheng YP. Reliability and validity of Hamilton depression scale assesses in 329 Chinese depression patients. Chin Ment Health J 1992;5:214-216

20. Cleary P, Guy W. Factor analysis of the Hamilton depression scale. Drugs Exp Clin Res 1977;1:115-120.

21. Williams JB. A structured interview guide for the Hamilton depression rating scale. Arch Gen Psychiatry 1988;45:742-747.

22. Lee C, Doo E, Choi JM, et al. The increased level of depression and anxiety in irritable bowel syndrome patients compared with healthy controls: systematic review and meta-analysis. J Neurogastroenterol Motil 2017;23:349-362.

23. Lackner JM, Ma CX, Keefer L, et al. Type, rather than number, of mental and physical comorbidities increases the severity of symptoms in patients with irritable bowel syndrome. Clin Gastroenterol Hepatol 2013;11:1147-1157.

24. Lin CH, Wang FC, Lin SC, Chen CC, Huang CJ. A comparison of inpatients with anxious depression to those with nonanxious depression. Psychiatry Res 2014;220:855-860.

25. Talley NJ, Phillips SF, Bruce B, Twomey CK, Zinsmeister AR, Melton LJ 3rd. Relation among personality and symptoms in nonulcer dyspepsia and the irritable bowel syndrome. Gastroenterology 1990;99:327-333.

26. Rey E, García-Alonso MO, Moreno-Ortega M, Alvarez-Sanchez A, Diaz-Rubio M. Determinants of quality of life in irritable bowel syndrome. J Clin Gastroenterol 2008;42:1003-1009.

27. Schneider MA, Fletcher PC. 'I feel as if my IBS is keeping me hostage!' exploring the negative impact of irritable bowel syndrome (IBS) and inflammatory bowel disease (IBD) upon university-aged women. Int J Nurs Pract 2008;14:135-148.

28. Miller V, Hopkins L, Whorwell PJ. Suicidal ideation in patients with irritable bowel syndrome. Clin Gastroenterol Hepatol 2004;2:1064-1068.

29. Eugenio MD, Jun SE, Cain KC, Jarrett ME, Heitkemper MM. Comprehensive self-management reduces the negative impact of irritable bowel syndrome symptoms on sexual functioning. Dig Dis Sci 2012;57:16361646.

30. Tu Q, Heitkemper MM, Jarrett ME, Buchanan DT. Sleep disturbances in irritable bowel syndrome: a systematic review. Neurogastroenterol Motil 2017;29:e12946.

31. Kanazawa M, Palsson OS, Thiwan SI, et al. Contributions of pain sensitivity and colonic motility to IBS symptom severity and predominant bowel habits. Am J Gastroenterol 2008;103:2550-2561.

32. Farabaugh AH, Bitran S, Witte J, et al. Anxious depression and early changes in the HAMD-17 anxiety-somatization factor items and antidepressant treatment outcome. Int Clin Psychopharmacol 2010;25:214217.

33. Howland RH, Wilson MG, Kornstein SG, et al. Factors predicting reduced antidepressant response: experience with the SNRI duloxetine in patients with major depression. Ann Clin Psychiatry 2008;20:209-218.

34. Spiegel DR, Kolb R. Treatment of irritable bowel syndrome with comorbid anxiety symptoms with mirtazapine. Clin Neuropharmacol 2011;34:36-38.

35. Drossman DA, Tack J, Ford AC, Szigethy E, Törnblom H, Van Oudenhove L. Neuromodulators for functional gastrointestinal disorders (disorders of gut-brain interaction): a Rome foundation working team report. Gastroenterology 2018;154:1140-1171, e1.

36. Gorard DA, Libby GW, Farthing MJ. Effect of a tricyclic antidepressant on small intestinal motility in health and diarrhea-predominant irritable bowel syndrome. Dig Dis Sci 1995;40:86-95. 